\title{
Magnetoplasmonic nanostructures: systems supporting both plasmonic and magnetic properties
}

\author{
G Armelles, A Cebollada, A García-Martín*, J M García-Martín, M U González, \\ J B González-Díaz, E Ferreiro-Vila and J F Torrado \\ Instituto de Microelectrónica de Madrid, IMM (CNM-CSIC), ClIsaac Newton 8 \\ (PTM). 28760-Tres Cantos (Madrid), Spain \\ *E-mail: antonio@imm.cnm.csic.es
}

\begin{abstract}
We present in this work our current understanding on magnetoplasmonic structures, that is, systems whose constituents exhibit simultaneously magnetic and plasmonic properties. We analyze both the influence of the plasmon resonance on the magneto-optical properties of the system and the ability of the magnetic field to modulate the plasmon properties. In particular we show how, in magnetoplasmonic systems sustaining localized or propagating surface plasmons, the associated electromagnetic field enhancement gives rise to an enhancement of the magneto-optical activity. On the other hand, we have analyzed the modulation of the propagating surface plasmon polariton wavevector in noble metal/ferromagnet/noble metal trilayers by an external magnetic field. These phenomena can be addressed as new concepts for the development of active plasmonic devices.
\end{abstract}

Keywords: surface plasmons, plasmonics, magneto-optics, nanophotonics. PACS:73.20.Mf, 78.20.Ls, 78.66.Bz, 78.67.Bf, 42.25.Bs

\section{Introduction}

During the last decades we have witnessed the development and fast growth of a new branch in optics: plasmon optics or plasmonics [1,2]. Surface plasmons (SP) are electromagnetic modes localized at the interface between two media with permittivity of opposite sign, such as those formed by a dielectric and a metal. They can exist in the form of propagating waves for extended metal films, the surface plasmon polariton (SPP) [3], or as localized resonances in small metallic particles, the localized surface plasmon (LSP) [4]. The application field of plasmon optics has become very broad, covering from the development of biosensors [5,6] or the design of optical nanoantennas [7] to the enhanced optical transmission on nanoperforated films [8]. In particular, by exploiting the confinement of SP to the metal-dielectric interface and their ability to overcome the diffraction limit, they are suitable candidates for the development of miniaturized optical (plasmonic) circuits [9]. However, in order to find new applications other than purely optical interconnections, it is necessary to add active functionalities to these SP-based devices, an area known as active plasmonics. The development of active plasmonics requires the use of materials or strategies in which the surface plasmon properties can be controlled by an external agent. To date, the feasibility of realization of active plasmonic devices, although still with limited applications, has been demonstrated by considering different control agents: temperature [10,11], electric field [12], or electromagnetic waves [13,14]. Another interesting candidate for being used as external agent, which we discuss in this paper, is the magnetic field.

The magnetic field ability to affect the properties of surface plasmon polaritons in highly doped InSb semiconductors is already known [15]. This effect is governed by the non-diagonal elements of the dielectric tensor, $\varepsilon_{\mathrm{ij}}$, responsible for the magneto-optical (MO) activity and whose value is controlled by the magnetic field, which in this kind of semiconductors turn out to be relatively high under the presence of moderate magnetic fields. However, for noble metals, the ones usually 
employed in plasmonics, extremely high magnetic fields (tens of Tesla) would be needed to obtain $\varepsilon_{\mathrm{ij}}$ elements with a sizeable value, and therefore enough SPP modulation properties. Ferromagnetic metals, on the contrary, possess large MO activity, associated with their magnetization, and could be suitable elements for active plasmonics. Nevertheless, ferromagnetic metals present an important drawback in their high absorption, which implies that the associated plasmon resonances are too broad and the propagation losses happen to be too high. A feasible way to reduce such damping without losing MO activity is to combine noble and ferromagnetic metals, forming what we denote as a magnetoplasmonic system. By combining both kinds of materials in smart structures we intend to obtain systems which simultaneously exhibit fair plasmon resonances and large enough MO activity, so that their SP properties are suitable for plasmon optics and can be modulated by means of a magnetic field.

Moreover, we can also consider the reciprocal MO-plasmon interaction, that is, the effect that SP excitation has on the MO response in a magnetoplasmonic system. Within this context, a strong enhancement of the Kerr rotation and ellipticity has been already predicted in nanoparticle metallic layers due to the localized surface plasmon resonances of the nanoparticles [16]. Again here, when these studies focus on noble metals, high magnetic fields are needed to observe the predicted phenomena, and this value can be reduced when using instead ferromagnetic metals with large MO activity. For example, a strong reduction of the MO activity has been observed in nanoperforated Co films in the spectral region corresponding to the anomalous optical transmission $[17,18]$. On the other hand, a strong enhancement of such MO activity has been observed in Co nanoparticles and arrays of Ni nanowires, which has been attributed to the localized surface plasmon resonances of the metallic nanostructures [19-21]. All these effects are however highly damped because of the high absorption losses of the ferromagnetic metals, as mentioned above, which makes again the magnetoplasmonic systems an ideal system to both analyze the influence of the SP modes on the MO response and to design configurations to maximize this effect.

In this paper, we present our current understanding on the phenomenology exhibited by magnetoplasmonic structures, analyzing their magneto-optical properties, their plasmonics characteristics and the intertwined effects between them. By the detailed analysis of three different configurations, we will show how in such systems it is possible both to enhance the MO activity of the system via surface plasmon excitation, and to modulate the plasmon properties via application of a magnetic field.

The first system under study consist of $\mathrm{Au} / \mathrm{Co} / \mathrm{Au}$ nanodiscs [22], where we will show how the LSP excitation leads to an enhancement of the electromagnetic field within the MO active layer, which in turns produces an enhancement of the MO activity of the system when compared to the continuous $\mathrm{Au} / \mathrm{Co} / \mathrm{Au}$ layer. The same influence of LSP can be observed in systems where the constituents responsible for plasmon excitation and MO activity are spatially detached. This will be shown in the second kind of examined structures, formed by $\mathrm{Au}$ nanodiscs and $\mathrm{Au} / \mathrm{Co} / \mathrm{Au}$ continuous trilayers separated by a dielectric spacer [23]. Here the LSP excitation on the nanodiscs induces a redistribution of the electromagnetic field at the Co layer, and an enhanced MO activity occurs at those energies where the amount of electromagnetic field in the magnetic layer is increased. Finally, the third kind of structures will consist of continuous $\mathrm{Au} / \mathrm{Co} / \mathrm{Au}$ and $\mathrm{Ag} / \mathrm{Co} / \mathrm{Ag}$ trilayers [24,25]. In these systems, the MO activity upon plasmon excitation is more than an order of magnitude higher compared to that without plasmon excitation. This enhancement is due to two effects: A first one is of purely optical origin, associated with the decrease of reflectivity of the system due to the SPP excitation and the subsequent electromagnetic field enhancement in the magneto-optically active layer. The second one is related to the modification of the SPP wavevector by the magnetic field, which consequently changes the SPP coupling conditions. This magnetic field induced SPP wavevector modulation indicates that this kind of magnetoplasmonic systems are potential candidates for the development of active (magnetic field controlled) plasmonic devices. 
Two MO effects will be considered in this work, both in reflection configuration. In the polar Kerr configuration the magnetization is perpendicular to the surface of the sample (figure 1(a)), and the variation of the polarization state (rotation, $\theta$ and ellipticity, $\phi$ ) when a linearly polarized light beam at normal incidence is reflected is measured. In this configuration one is sensitive to the "sample-plane" (xy) elements of the dielectric tensor. The complex Kerr rotation $(\Phi=\theta+i \phi)$ equals $r_{p s} / r_{p p}$, where $r_{p s}$ reflects the polarization conversion upon reflection induced by the MO properties and $r_{p p}$ is the "purely" optical complex reflection. In the other configuration, the transverse Kerr effect (figure 1(b)), the sample magnetization lies in the sample plane and perpendicular to the plane of incidence of the light, and the relevant magnitude is the change in reflectivity of p-polarized light when the magnetization is reversed, $\Delta \mathrm{R} / \mathrm{R}$. In this case, the measurement is sensitive to the "incidence-plane" (xz) elements of the dielectric tensor.

(a)

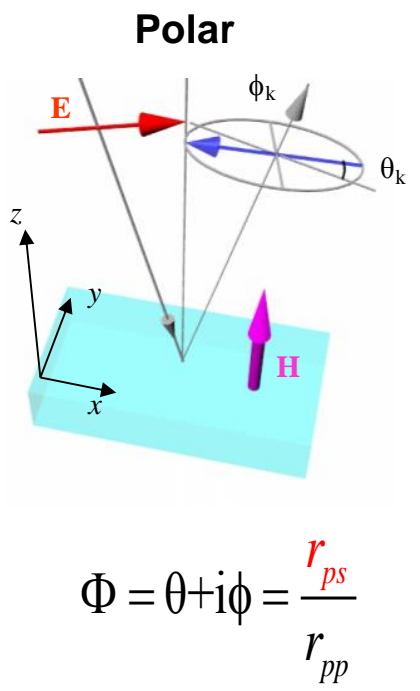

(b)

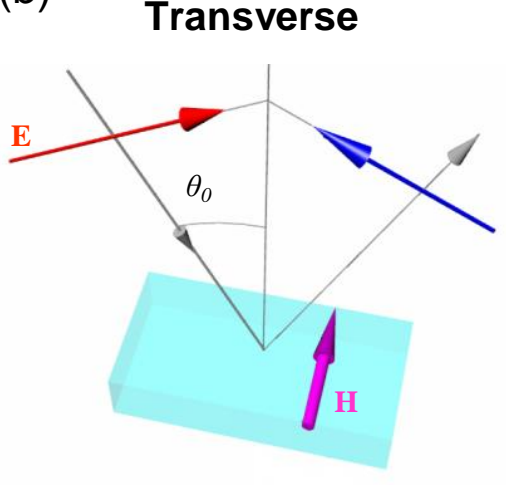

$$
\boldsymbol{\varepsilon}=\left(\begin{array}{ccc}
\varepsilon_{x x} & \varepsilon_{x y} & 0 \\
-\varepsilon_{x y} & \varepsilon_{x x} & 0 \\
0 & 0 & \varepsilon_{z z}
\end{array}\right)
$$

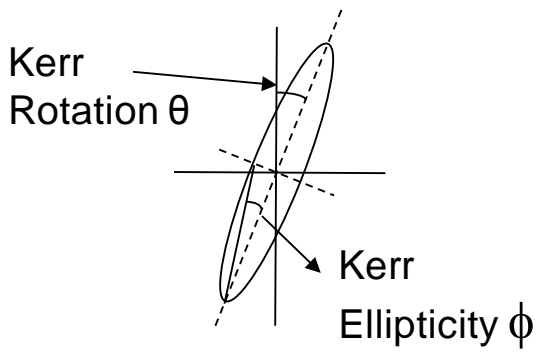

$$
\begin{gathered}
\boldsymbol{\varepsilon}=\left(\begin{array}{ccc}
\varepsilon_{x x} & 0 & \varepsilon_{x z} \\
0 & \varepsilon_{x x} & 0 \\
-\varepsilon_{x z} & 0 & \varepsilon_{z z}
\end{array}\right) \\
\frac{\Delta \mathrm{R}}{\mathrm{R}}=\frac{\mathrm{R}_{\mathrm{pp}}(+\mathrm{H})-\mathrm{R}_{\mathrm{pp}}(-\mathrm{H})}{2 \mathrm{R}_{\mathrm{pp}}(0)}
\end{gathered}
$$

Figure 1. Scheme of the geometry and the relevant magnitudes for the two magneto-optical effects considered in this work: (a) polar Kerr configuration; (b) transverse Kerr configuration.

\section{LSP effects on the MO activity in Au/Co/Au nanodiscs}

As mentioned, the first system considered in this study consists of nanodiscs of $\mathrm{Au} / \mathrm{Co} / \mathrm{Au}$, deposited on top of glass substrates, sustaining both MO activity and localized surface plasmons. The nanodiscs have been obtained by means of colloidal lithography [26-28] from sputtered $\mathrm{Au} / \mathrm{Co} / \mathrm{Au}$ trilayer films. 
The composition of the films was kept constant $(6 \mathrm{~nm} \mathrm{Au} / 10 \mathrm{~nm} \mathrm{Co} / 16 \mathrm{~nm} \mathrm{Au/glass})$, and the nanodiscs size was controlled by using latex spheres with different diameter $(60,75$ and $110 \mathrm{~nm})$. More details on the sample fabrication can be found in ref. [22]. Figures 2(a) and (b) show atomic force microscopy (AFM) images of the nanodiscs obtained using latex spheres of 60 and $110 \mathrm{~nm}$ diameters, respectively.

The plasmonic properties of the nanodiscs have been characterized by conventional UV/Vis extinction spectroscopy. Figure 2(c) shows the obtained extinction spectra for the nanodiscs of 60,76 and $110 \mathrm{~nm}$ diameter. As can be observed, the spectra of all the samples are characterized by a peak associated with the LSP resonance whose position red-shifts as the disc diameter is increased, in agreement with that observed for pure noble metal nanoparticles. To highlight the effect of the incorporation of Co in the nanodiscs, we show in figure 2(d) the calculated extinction spectra of

(a)

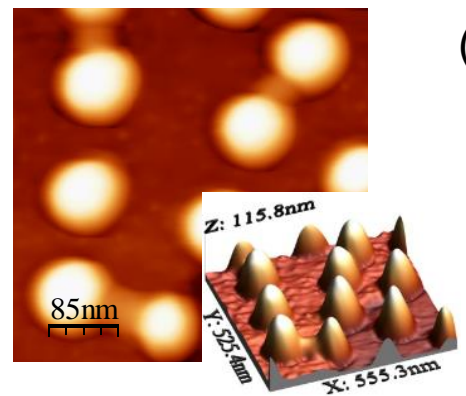

(b)
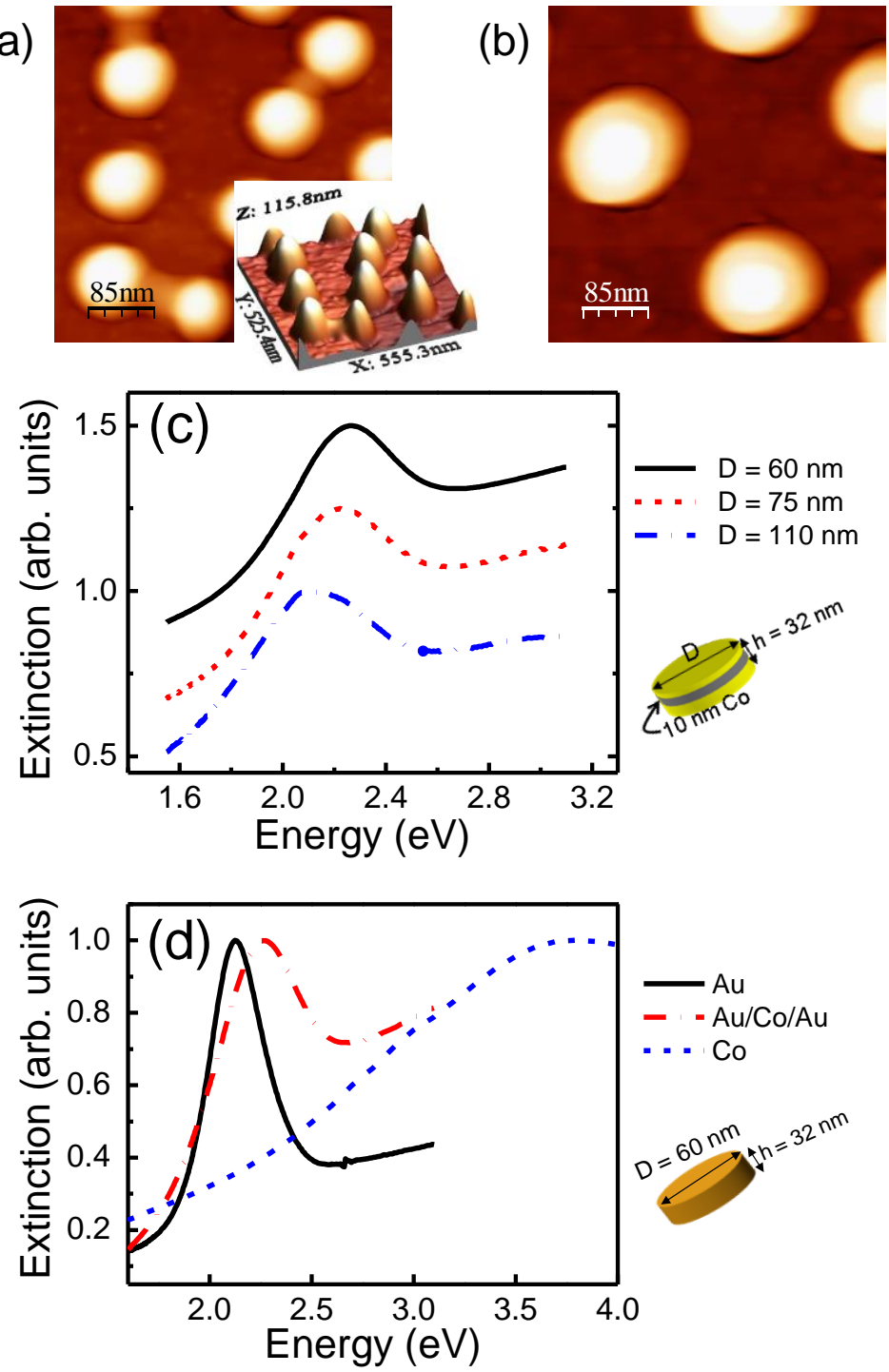

Figure 2. (a), (b) AFM image of Au/Co/Au nanodiscs (6 nm Au/10 nm Co/16 nm Au) of $60 \mathrm{~nm}$ and $110 \mathrm{~nm}$ diameter, respectively. (c) Experimental extinction spectra of $\mathrm{Au} / \mathrm{Co} / \mathrm{Au}$ nanodiscs of different diameters: $\mathrm{D}=60,75$ and $110 \mathrm{~nm}$. (d) Simulated extinction spectra of nanodiscs composed of pure noble metal, pure ferromagnetic metal and the magnetoplasmonic trilayer. All nanodots have $60 \mathrm{~nm}$ diameter and a total height of $32 \mathrm{~nm}$. 
similar nanodiscs of $60 \mathrm{~nm}$ diameter and $32 \mathrm{~nm}$ total metal height whose composition have been varied from pure $\mathrm{Au}$ or pure $\mathrm{Co}$ to the $\mathrm{Au} / \mathrm{Co} / \mathrm{Au}$ trilayer configuration. As can be clearly observed in this figure, the Co nanodiscs have a much broader and shifted towards the blue LSP resonance than those made of pure $\mathrm{Au}$, due to the optical properties of Co (high absorption and negative real part of the dielectric constant well in the UV). In the trilayer nanodiscs, where a thin layer of $\mathrm{Co}$ is incorporated, the LSP peak properties are mainly determined by the Au but also affected by the optical properties of the Co layer (the LSP associated electromagnetic field is feeling this layer too) and as a consequence it is slightly broader than that of pure Au nanodiscs and blue-shifted.

Moreover, the effect of incorporating Co into the nanodiscs is also to introduce MO activity on the system. The $\mathrm{MO}$ characterization of these $\mathrm{Au} / \mathrm{Co} / \mathrm{Au}$ nanodiscs has been performed by means of Kerr spectroscopy in the polar configuration. The sample, placed inside an electromagnet that applies a magnetic field perpendicular to its surface, is illuminated at normal incidence by a monochromatic beam coming from a Xe lamp followed by a monochromator. By modulating the beam polarization with a photoelastic modulator, the Kerr rotation and ellipticity for each wavelength are determined [29]. In figure 3(a) we show a typical ellipticity versus magnetic field loop (hysteresis loop of the Kerr ellipticity) for the $60 \mathrm{~nm}$ diameter nanodiscs at normal incidence and at a fixed wavelength. As the magnetic field is increased, the Kerr ellipticity increases and reaches a saturation value for a magnetic field of around 12000 Oe. This value corresponds to the magnetic field needed to saturate the magnetization of the Co layer of the nanodiscs. (The particular shape of the hysteresis loop, very inclined, is due to the fact that in these samples the axis perpendicular to the surface normal is a hard magnetization axis.) The value of the ellipticity at saturation depends on the wavelength of the incident light, as is shown in Figure 3(b), where we present its wavelength dependence for nanodiscs of 60 and $110 \mathrm{~nm}$ diameters compared to that of a continuous $\mathrm{Au} / \mathrm{Co} / \mathrm{Au}$ layer with identical $\mathrm{Au}$ and Co thicknesses. Remarkably, the nanodiscs Kerr ellipticity spectra present peaks at the same energetic region as the extinction peak corresponding to LSP excitation. Moreover, the position of the maximum of the Kerr ellipticity is red-shifted as the size of the nanodiscs is increased, in agreement with the shift in the extinction peak. This allows us to associate the modification of the MO response of the nanostructures as compared to the continuous film to the presence of the LSP resonance. Furthermore, although the amount of $\mathrm{Co}$ in the continuous layer is nearly five times larger than that of the nanodisc layer, the magnitude of the MO effect is similar, which indicates that the LSP present in the nanoparticles lead to a large enhancement of the MO activity. This enhancement is more clearly visualized in figure 3(d), where we plot the total Kerr activity $\Phi^{2}$ of the 60 and $110 \mathrm{~nm}$ diameter nanodiscs normalized to that of a continuous $\mathrm{Au} / \mathrm{Co} / \mathrm{film}$ with the same composition and amount of material (i.e. multiplied by the filling fraction $f$ ).

To further understand the origins of the MO effects observed and their correlation with the localized surface plasmons, we calculated the polar Kerr ellipticity for an array of $\mathrm{Au} / \mathrm{Co} / \mathrm{Au}$ nanodiscs with identical dimensions to those studied experimentally. This was done using a scattering matrix formalism adapted to treat materials with MO activity that allows an exact solution for the wave propagation in ordered arrays [30]. The formalism took into account all the interactions between the different nanoparticles as well as the substrate effects. The nanodiscs were modelled as sandwiches of three discs with diameters equal to those of the experimental nanodiscs. We have considered two types of geometries for the arrangement of the discs: triangular and square. The distance between the discs (lattice parameter) is determined by the surface coverage of the polystyrene spheres. The optical properties of the $\mathrm{Au}$ and $\mathrm{Co}$ that were used came from reference [24] and the MO constants of Co were extracted from the polar Kerr rotation and ellipticity spectra for the continuous $\mathrm{Au} / \mathrm{Co} / \mathrm{Au}$ film. Figure 3(c) shows the theoretical results for the continuous $\mathrm{Au} / \mathrm{Co} / \mathrm{Au}$ film and for the 60 and $110 \mathrm{~nm}$ diameter systems only in the triangular arrangement for clarity, since, for this coverage, the results obtained for the square arrangement do not present any noticeable difference. Both the presence of a peak in the Kerr ellipticity spectra at those energies where the LSP peak appears and its red-shift when the disc diameter increases are reproduced. The enhancement of the MO activity compared to that of 

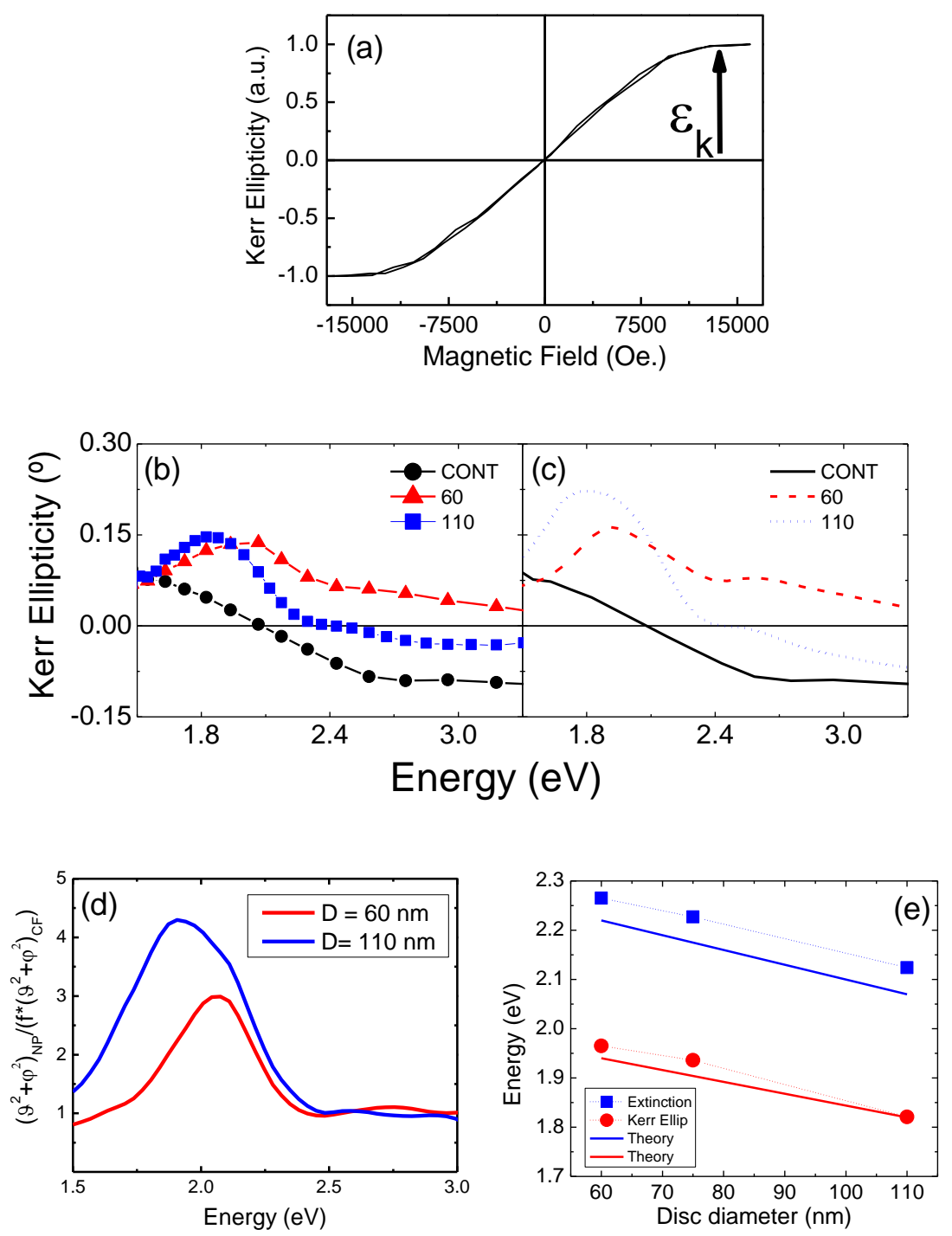

Figure 3. (a) Ellipticity hysteresis loop for $60 \mathrm{~nm}$ diameter discs. (b) Experimental, and (c) calculated, Kerr ellipticity spectra for nanoparticles of two different diameters: 60 and $110 \mathrm{~nm}$. The spectrum of a continuous $\mathrm{Au} / \mathrm{Co} / \mathrm{Au}$ film with the same layer thicknesses is included for comparison. (d) Total polar Kerr activity for $\mathrm{Au} / \mathrm{Co} / \mathrm{Au}$ nanodiscs with 60 and $110 \mathrm{~nm}$ diameter, normalized to that of a continuous film with the same amount of material. (e) Position of the extinction and Kerr ellipticity peaks as a function of the nanodiscs size. Dots and lines represent experimental and theoretical positions, respectively.

the continuous film also appears, although the values for the theoretical spectra were higher than the experimental ones. This effect was attributed to an overestimation of the active amount of Co inside the nanoparticles. This reduction could arise from a slight oxidation of the Co on the lateral of the disc since it was exposed to air [31].

We have also calculated the extinction spectrum of the discs layer and, in figure 3(e), we present the theoretical position of the extinction and ellipticity peaks as a function of the disc diameter. The theoretical results reproduced the trend observed for the experimental results: a red-shift of both peaks as we increase the size of the nanoparticles and a red-shift of the position of the ellipticity peak with respect to the absorption peak. Therefore, we can conclude that the structure observed in the polar 
Kerr spectra was indeed related to the LSP of the $\mathrm{Au} / \mathrm{Co} / \mathrm{Au}$ nanoparticles. The plasmon excitation produces an enhancement of the electromagnetic field located in the MO active material of the system (the Co layer) giving rise to the overall MO activity enhancement. The red-shift of the position of the ellipticity peak with respect to that of the extinction peak could result from the different wavelength dependence of the optical and MO constants.

\section{LSP effects of Au nanodiscs in the MO activity of Au/Co/Au continuous films}

The analysis of the $\mathrm{Au} / \mathrm{Co} / \mathrm{Au}$ nanodiscs has demonstrated that SP excitations modify and enhance the MO activity of the system. However, in principle it is not necessary that the surface plasmon responsible for this enhanced MO activity occurs in intimate contact with the ferromagnetic layer. In fact, the enhancement is expected to remain as long as the evanescent field of the plasmon excitation extends long enough to reach the MO active layer. To demonstrate this hypothesis, we proceed now to the analysis of a set of magnetoplasmonic structures in which the MO active component and the plasmonic excitation are spatially detached. The MO active region, consisting of a $6 \mathrm{~nm} \mathrm{Au} / 10 \mathrm{~nm}$

(a)
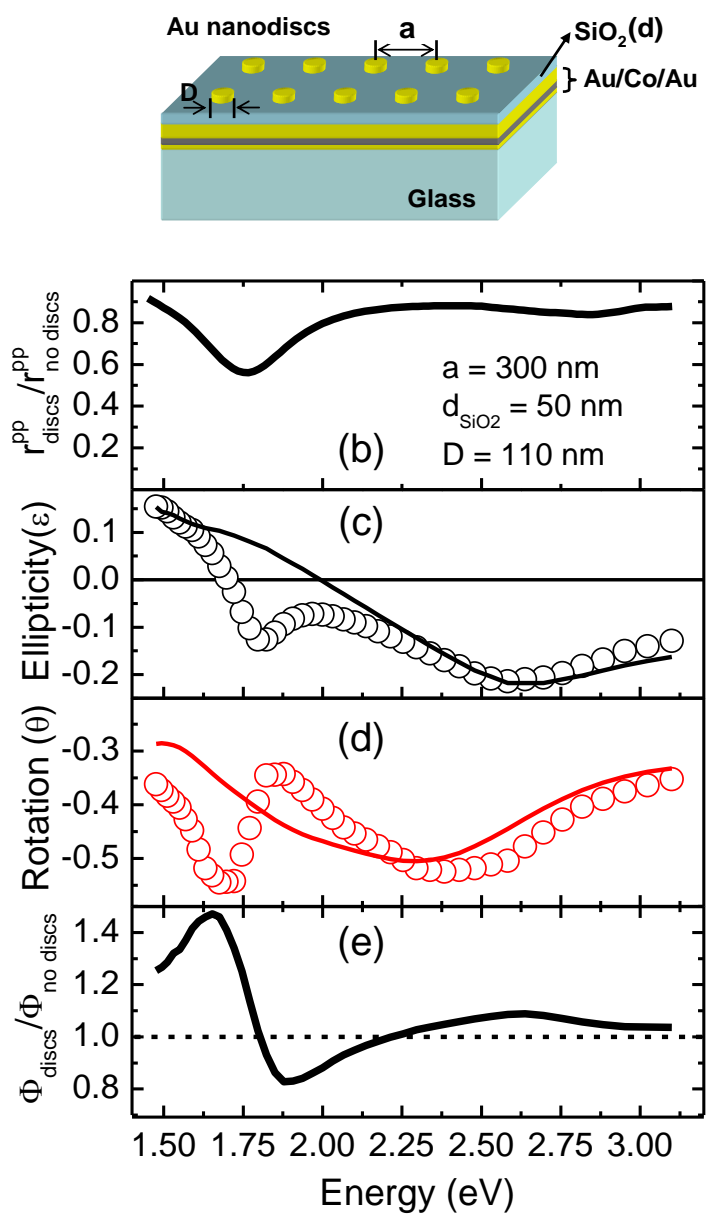

Figure 4. (a) Schema of the system with magneto-optical activity and localized surface plasmon resonance spatially detached. (b) Reflectivity spectrum of the system showing the dip associated with the nanodiscs localized surface plasmon resonance. (c) Kerr ellipticity- and (d) Kerr rotationfor the system (dots). The solid lines correspond to the same magnitudes for the continuous trilayer without dots on top. (e) Complex polar Kerr rotation of the system. 


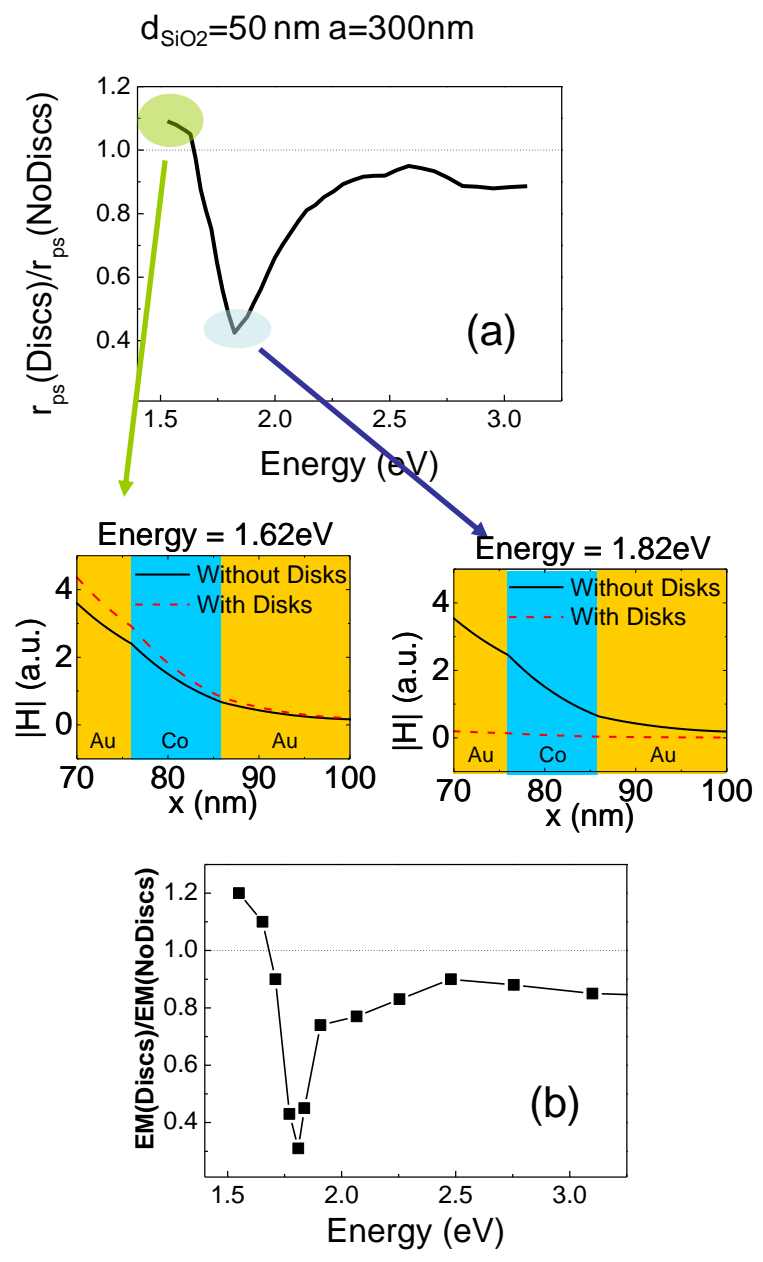

Figure 5. (a) $r_{p s}$ component of the reflectivity for the $\mathrm{Au} / \mathrm{Co} / \mathrm{Au}$ trilayer $/ \mathrm{SiO}_{2} / \mathrm{Au}$ nanodiscs system, as calculated from the complex polar Kerr rotation, and calculated electromagnetic (EM) field distributions at the energies where $r_{p s}$ is maximum and minimum. (b) Ratio of the EM field inside the trilayer with and without discs on top.

$\mathrm{Co} / 16 \mathrm{~nm} \mathrm{Au}$ continuous trilayer grown by magnetron sputtering, is separated from a squared array of Au nanodiscs (110 nm diameter and $20 \mathrm{~nm}$ thick) by a thin $\mathrm{SiO}_{2}$ layer (see figure 4(a)) [23].

The reflectivity spectrum shown in figure 4(b) for a structure with a lattice constant $a$ of $300 \mathrm{~nm}$, normalized to the signal through a $\mathrm{SiO}_{2} / \mathrm{Au} / \mathrm{Co} / \mathrm{Au}$ region without discs, presents a single dip at around $1.75 \mathrm{eV}$, corresponding to the Au nanodiscs LSP resonance excitation. As in the previous case the following step is to obtain polar Kerr ellipticity and rotation spectra (figures 4(c) and (d)). Again clear effects in the spectral region where the LSP resonance is excited can be seen when compared with equivalent layers without Au nanodiscs. These are characterized by a narrow dip in the Kerr ellipticity around $1.8 \mathrm{eV}$ and an s-like structure in the Kerr rotation at the same energy region, both absent in the continuous layer structures. This s-like structure is also observed if the normalized complex polar Kerr rotation $\Phi$ spectra are computed from the corresponding rotation and ellipticity ones (figure 4(e)). Bearing in mind that this magnitude can be expressed as the fraction $r_{p s} / r_{p p}$, ie, purely magnetic and purely optical components, it is possible now to extract $r_{p s}$ and analyze possible effects of this component in addition to the optical ones concluded from $r_{p p}$. This is shown in figure $5(\mathrm{a})$, where the spectral dependence of $r_{\mathrm{ps}}$ normalized to that of the region without discs is presented. Again the results show strong energy dependence, with a normalized polarization conversion $r_{p s}$ larger 
than one at energies below the LSP resonance, and smaller than one at higher energies, exhibiting a minimum at $1.82 \mathrm{eV}$, pretty close to the LSP excitation energy. This can be clearly understood by inspecting the electromagnetic (EM) field intensity distribution inside the different layers at specific energy positions. As can be seen in figure 5(b), at energies below LSP excitation the electromagnetic field in the MO active layer (Co) is larger in the region with discs than in the region without discs, while the situation is reversed at energies corresponding to the LSP excitation. The presence of the resonant nanodiscs produces a different rearrangement of the EM field at different energies, and at those energies where the EM field is enhanced in the magnetic layer there is an increment of the purely magneto-optical contribution $r_{p s}$.

\section{SPP effects in the MO activity of $\mathrm{Au} / \mathrm{Co} / \mathrm{Au}$ and $\mathrm{Ag} / \mathrm{Co} / \mathrm{Ag}$ continuous films}

So far we have shown that the excitation of LSP resonances, either in $\mathrm{Au} / \mathrm{Co} / \mathrm{Au}$ nanodiscs or in $\mathrm{Au}$ discs close to continuous $\mathrm{Au} / \mathrm{Co} / \mathrm{Au}$ trilayers, leads to strong modifications of the MO activity of the system via modification of the optical response as well as of the EM field in the MO active layer. Here we will show that propagating SPPs may play a similar role in continuous $\mathrm{Au} / \mathrm{Co} / \mathrm{Au}$ trilayers. To excite the SPP we use the well known Kretschmann configuration (see figure 6(a) for a sketch) and we measure the transverse Kerr effect instead, to be able to use low magnetic fields and avoid the large Faraday signal of the prism that would appear if the polar Kerr configuration was used, in a similar fashion of the pioneer work of Hermann et al. [32]. The magnetic field is applied by means of an electromagnet coil placed directly above the sample. Two sets of magnetoplasmonic trilayers were grown, using $\mathrm{Au}$ and $\mathrm{Ag}$ as plasmonic materials. In the case of $\mathrm{Ag}$, a Pt capping layer was deposited to avoid chemical deterioration with time. The studied structures were $6 \mathrm{~nm} \mathrm{Au} / X \mathrm{Co} / 16 \mathrm{~nm} \mathrm{Au}$ trilayers grown by molecular beam epitaxy (MBE) on glass substrates with Co thickness $X$ varying between 1 and $10 \mathrm{~nm}$, and $2 \mathrm{~nm} \mathrm{Pt} / 7 \mathrm{~nm} \mathrm{Ag} / X$ Co/ $16 \mathrm{~nm} \mathrm{Ag} \mathrm{multilayers} \mathrm{grown} \mathrm{by} \mathrm{magnetron}$ sputtering, with Co thickness $X$ varying between 1 and $7 \mathrm{~nm}$. The average rms roughness of the $\mathrm{Au} / \mathrm{Co} / \mathrm{Au}$ layers is $1.5 \mathrm{~nm}$ [33]; for the $\mathrm{Pt} / \mathrm{Ag} / \mathrm{Co} / \mathrm{Ag}$ system the roughness rms value increases with the Co thickness ranging from $3 \mathrm{~nm}$ for $1 \mathrm{~nm}$ Co to $4 \mathrm{~nm}$ for $7 \mathrm{~nm}$ Co [25]. For these structures the optical and magnetooptical characterizations consist of measurements of reflectivity and transverse Kerr signal $\Delta R / R$ versus incident angle in the Kretschmann configuration at a wavelength of $632 \mathrm{~nm}$. Reflectivity presents a strong minimum when the SPP is excited, and in the $\Delta \mathrm{R} / \mathrm{R}$ curves similar resonance-like structures are visible in both cases, related to the excitation of the SPP (see figures 6(b) and $6(\mathrm{~d})$ ). The $\Delta \mathrm{R} / \mathrm{R}$ values around SPP excitation are one order of magnitude higher than those obtained at angles where no SPP is excited, or than those obtained when light impinges from the air side, where SPP excitation is not possible, showing again the enhancement of the MO activity induced by the surface plasmons. The $\Delta \mathrm{R} / \mathrm{R}$ curves are shown in the same figures as the optical counterpart (figures $6(\mathrm{~b})$ and $6(\mathrm{~d})$ ) to make clear two important points: (i) the $\Delta \mathrm{R} / \mathrm{R}$ signal has a zero crossing where the optical signal presents a minimum (this will be of importance in the following section); (ii) the slope of the $\Delta R / R$ signal is larger than that of the optical one, which is important for the development of high sensitivity MO surface plasmon resonance based biosensors [34].

If one now considers the evolution of the maximum value of the transverse Kerr signal $\left(\Delta R / R_{\text {Max }}\right)$ as a function of Co thickness (see figures 6(c) and 6(e)), a maximum is obtained at around $6 \mathrm{~nm}$ for the Au structures and around $2 \mathrm{~nm}$ for the Pt capped Ag ones. The Co thickness values which yield maximum MO activity correspond to the optimum excitation of the SPP and are correlated both with a reduction of the reflectivity of the system and with an enhancement in the magnetic field induced reflectivity change $(\Delta \mathrm{R})$ [24]. The difference in the total amount of $\Delta \mathrm{R} / \mathrm{R}$ as well as the large difference in the value of the Co thickness that optimizes the signal between the Au and Ag systems are due to the presence of the Pt capping layer, of absorbing character, whose effect is equivalent to adding 2 extra $\mathrm{nm}$ of Co but without MO activity. The $\Delta \mathrm{R} / \mathrm{R}$ values obtained without SPP excitation are also included in figures 6(c) and 6(e) for comparison. 
(a)

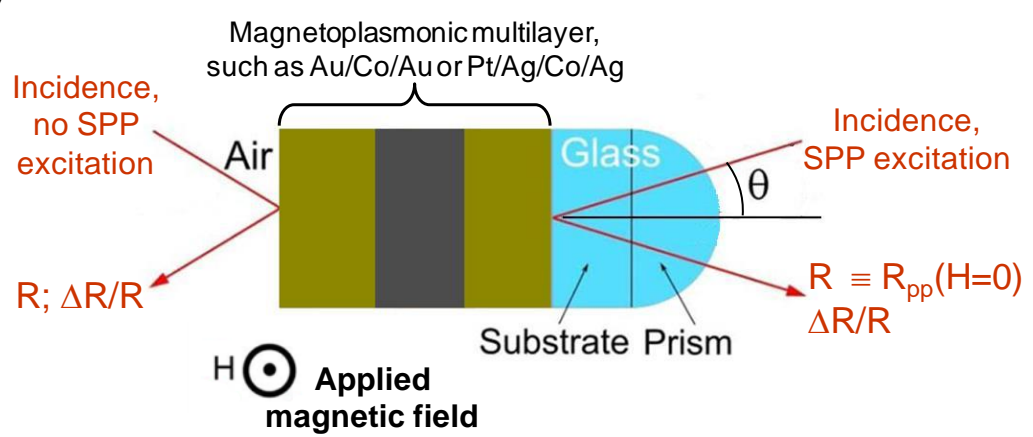

$\mathrm{Au} / \mathrm{Co} / \mathrm{Au}$

(b)

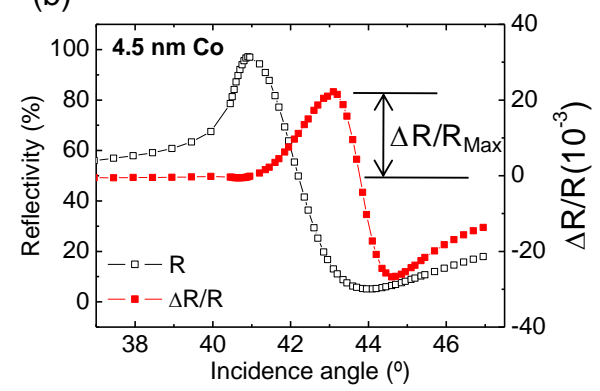

(c)

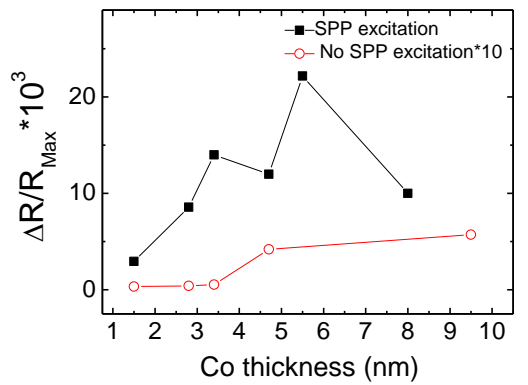

$\mathrm{Pt} / \mathrm{Ag} / \mathrm{Co} / \mathrm{Ag}$

(d)

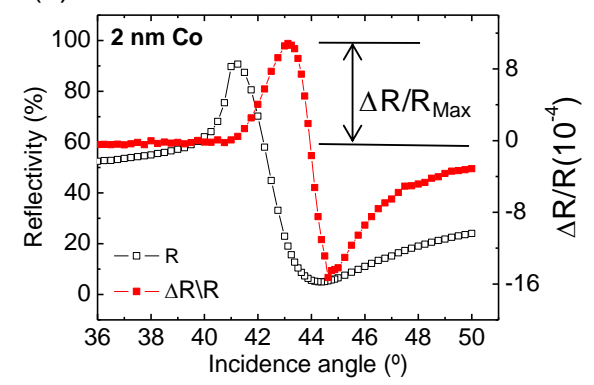

(e)

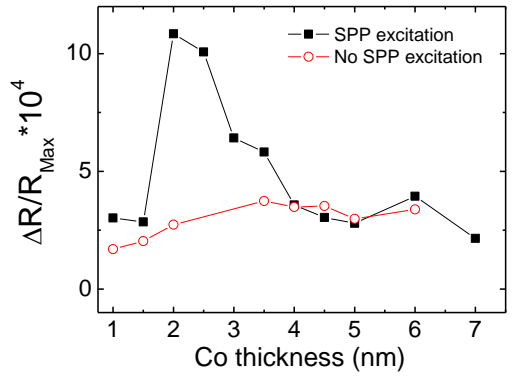

Figure 6. (a) Measurement configuration for the magnetoplasmonic continuous films. Reflectivity, $\mathrm{R}$, and transverse Kerr signal, $\Delta \mathrm{R} / \mathrm{R}$, are measured with incidence from the air side (excitation of SPP is not possible) and from the glass side in the Kretschmann-Raether configuration (allowing SPP excitation). (b) Reflectivity and $\Delta \mathrm{R} / \mathrm{R}$ for a $\mathrm{Au} / \mathrm{Co} / \mathrm{Au}$ trilayer measured with incidence through the glass prism. (c) Dependence of the maximum value of $\Delta R / R$ with the thickness of the Co layer for the $\mathrm{Au} / \mathrm{Co} / \mathrm{Au}$ trilayer, obtained upon and without SPP excitation. (d) Reflectivity and $\Delta \mathrm{R} / \mathrm{R}$ for a $\mathrm{Pt} / \mathrm{Ag} / \mathrm{Co} / \mathrm{Ag}$ multilayer under incidence from the glass side. (e) Evolution of the maximum value of $\Delta \mathrm{R} / \mathrm{R}$ with the thickness of the Co layer for the $\mathrm{Pt} / \mathrm{Ag} / \mathrm{Co} / \mathrm{Ag}$ films.

In a similar fashion to the study presented in section 3, the calculation of the EM field distribution allows further understanding of the observed effects. In figure 7 we show this parameter along the different layers, both when SPP excitation is not allowed (incidence from the air side) and allowed (incidence from the glass side). First, when no SPP is excited, the reflectivity versus angle curve exhibits, as expected, a monotonous behaviour (figure 7(a)). In the three selected angular positions, the corresponding EM field (figures 7(b)-(d)) gradually decreases from the incident light side (air) with an almost exponential behaviour. On the other hand, under SPP excitation conditions, a typical minimum in the reflectivity curve is obtained (figure 7(e)). The corresponding EM distribution also decreases 

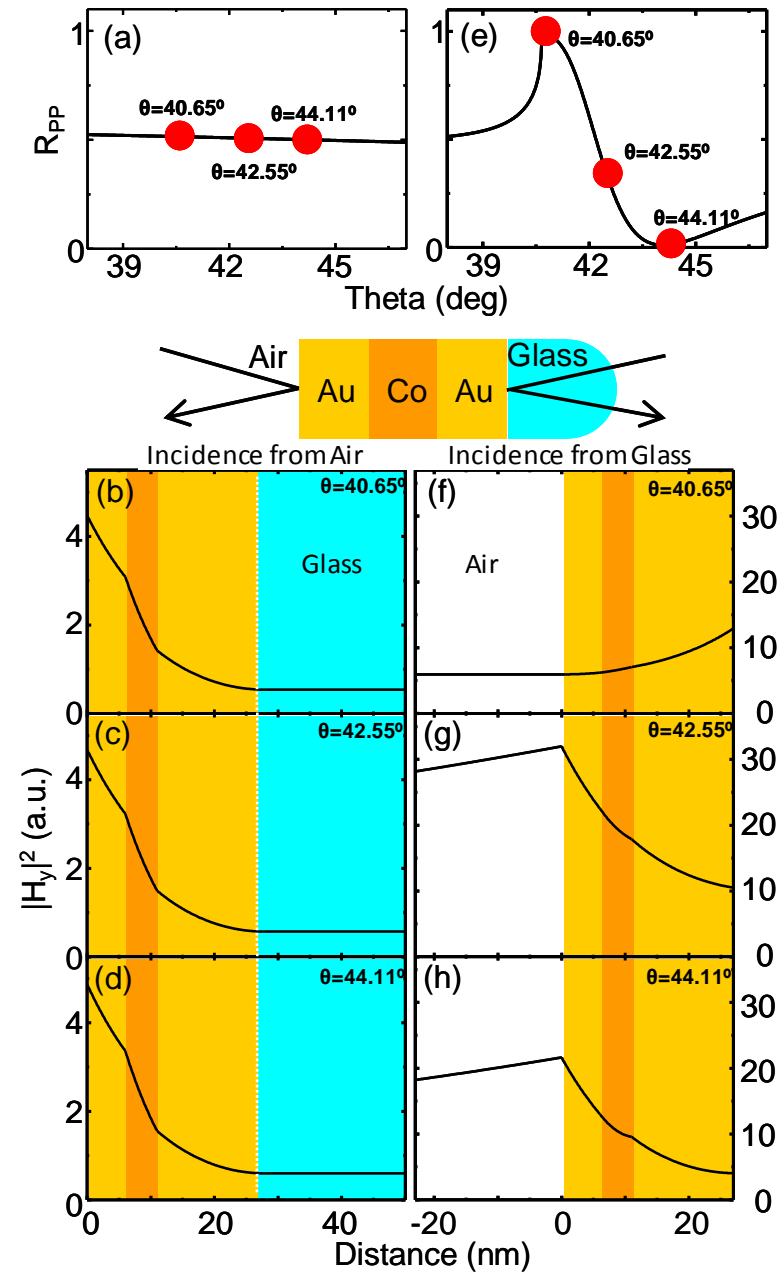

Figure 7. (a) Reflectivity with incidence from the air side (no SPP excitation), and (b) - (d) corresponding calculated electromagnetic field distribution for three given angles. (e) - (h) Same but with incidence from the glass side, with SPP excitation possible.

from the incident light side (glass) for the angular position corresponding to total reflection (figure $7(\mathrm{f})$ ), where the plasmon is not yet excited. However, for the other two selected angular positions (figures $7(\mathrm{~g})-(\mathrm{h})$ ), where the SPP has already been excited, the EM field gradually increases as the light gets deeper into the structure, reaching as expected a maximum value at the air-Au interface, where the SPP is located. This implies that for these two last cases the EM field is drastically enhanced at the MO active layer region (note the different vertical scales for both EM field axis), being responsible for the enhancement of the magnetooptical signal.

\section{Magnetic field modulation of the wave vector or SPP's in Au/Co/Au continuous films}

In the previous sections we have given ample proof of the influence of plasmon excitation, either LSP in discrete elements or SPP in continuous films, in the MO activity of magnetoplasmonic systems. As mentioned above, the plasmon-MO relationship is bidirectional, meaning that the presence of elements with MO activity makes the properties of the plasmon suitable to be altered by an external magnetic field. Such external modification capabilities are the requirements to go one step forward in the development of plasmonics or nanophotonics, since they imply that the elements are now active. To achieve that goal we need to be able to modify the propagation of the signal, this means controlling the 
value of the plasmon wavevector. In a very simple system, consisting of a very thin layer of ferromagnetic material at the metal-dielectric interface in the transverse configuration, and considering that the MO element $\varepsilon_{\mathrm{xz}}$ is much smaller than $\varepsilon_{\mathrm{xx}}$ one can obtain the modification of the plasmon wavevector $\mathrm{k}_{\mathrm{sp}}$ to the first order in $\varepsilon_{\mathrm{xz}}$ as:

$$
\frac{\Delta k_{s p}}{k_{s p}} \equiv \frac{k_{s p}( \pm H)-k_{s p}(0)}{k_{s p}(0)}=\frac{2 i}{\varepsilon_{x x}^{2}-1} \sqrt{\frac{\varepsilon_{x x}}{1+\varepsilon_{x x}}} \frac{2 \pi d}{\lambda}\left( \pm \varepsilon_{x z}\right)
$$

The expression implies the MO modulation of the plasmon wavevector to increase linearly as a function of the thickness of the magnetic layer. Unfortunately, absorption losses would also monotonically increase as the layer thickness grows. The modulation is also proportional to the non-diagonal element of the dielectric tensor, thus the use of materials with high values at low magnetic fields is required.

For an actual example we are going to consider the set of $\mathrm{Au} / \mathrm{Co} / \mathrm{Au}$ films described in the preceding section. Reflection and transverse Kerr signal as a function of the incident angle are depicted in figure 6(b). The transverse Kerr signal is obtained through the modulation of the reflectivity induced by the magnetic field, but this magnetic field modulation can be also seen as a modulation in the plasmon wavevector. Moreover, a variation of the plasmon wavevector implies a variation of the angle at which the light-plasmon coupling takes place, so we end up with the fact that magnetic modulation turns into angular modulation. That explains the shape of the Kerr signal in figure 6(b), since it can directly be related to the angular derivative of the reflectivity signal as [24]:

$$
\left.\frac{\Delta R_{p p}}{R_{p p}}\right|_{\text {Mag }}=\frac{\partial R}{\partial \theta} \frac{\Delta \theta}{R_{p p}}
$$

From this expression one can experimentally obtain the value of $\Delta \theta$ for SPP coupling associated with the magnetic field contribution by comparing the transverse Kerr signal and the angular derivative of the reflectivity, as depicted in figure 8(a), where such comparison is made for a trilayer with a Co thickness of $4.5 \mathrm{~nm}$. From the value of $\Delta \theta$ it is easy to obtain the experimental value of $\Delta \mathrm{k}_{\mathrm{sp}} / \mathrm{k}_{\mathrm{sp}}=\operatorname{cotg}(\theta) \Delta \theta$. In figure 8(b) we have depicted the value of $\Delta \mathrm{k}_{\mathrm{sp}} / \mathrm{k}_{\mathrm{sp}}$ thus obtained (dots) together with the theoretical ones extracted from the shift in the reflectivity curves under magnetic field (lines). As can be seen the modulation of $\mathrm{k}_{\mathrm{sp}}$ increases monotonically as a function of the Co thickness as expected from Eq. 1, although not linearly due to the complexity of the actual system.

\section{Summary and conclusions}

We have shown how the combination of ferromagnetic and plasmonic materials allows designing structures whose $\mathrm{MO}$ and plasmonic properties can be controlled.

In systems exhibiting LSP and SPP resonances, their excitation leads to important optical properties modifications and EM field redistribution which in turn produces strong modifications of the overall MO response of the system. This has been observed in nanodisc layers, continuous films and the combination of both, and even exploring different plasmonic components such as Au and Ag. The enhancement of the MO activity of such systems opens new routes for the development of novel MO suface plasmon resonance sensors of higher sensitivity.

On the other hand, the presence of the ferromagnetic material makes that the wavevector of SPP waves propagating in the magnetoplasmonic structures can be modulated by the application of an external magnetic field in sizeable values. In this way, the magnetoplasmonic systems are potential candidates for the development of active plasmonics devices, key factor in the design of plasmonbased telecommunication applications. 

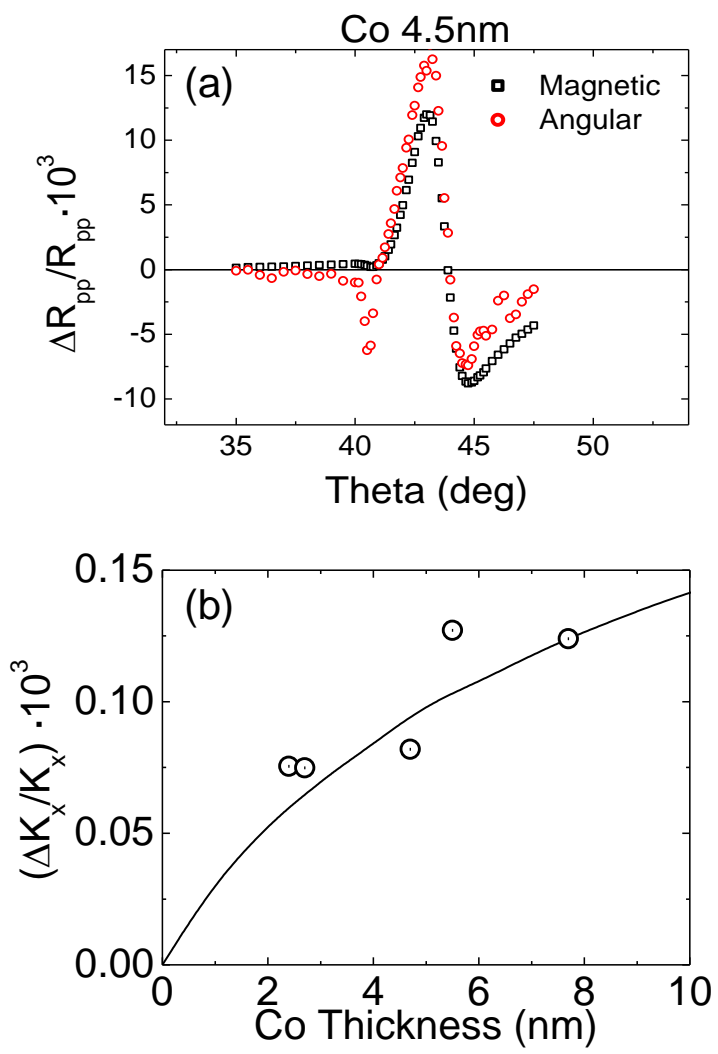

Figure 8. (a) Transverse magneto-optical Kerr signal, $\Delta R / R$, and angular derivative of the reflectivity upon SPP excitation, for a $\mathrm{Au} / \mathrm{Co} / \mathrm{Au}$ continuous trilayer. (b) Evolution of the modulation of the SPP wavevector by the magnetic field with the Co layer thickness.

\section{Acknowledgments:}

We acknowledge our collaborators C Clavero, R A Lukaszew, J R Skuza, D P Kumah, R Clarke, B Sepúlveda, Y Alaverdyan, M Käll, S Acimovic, J Cesario, R Quidant, G Badenes, X M Bendaña Sueiro, D Meneses Rodríguez and E Muñoz Sandoval.

We also acknowledge financial support from Spanish Ministry of Science and Education (NAN200409195-C04, MAT2005-05524-C02-01, "FUNCOAT" CONSOLIDER INGENIO 2010 CSD200800023, "MAGPLAS" MAT2008-06765-C02-01/NAN), Comunidad de Madrid (S-0505/MAT/0194 and Microseres), CSIC (Refs. 200650I130 and 200650I032) and UE (NoE-Phoremost and "NANOMAGMA" EU NMP-FP7-214107).

\section{References}

[1] Maier S A 2007 Plasmonics: Fundamentals and Applications (Berlin: Springer)

[2] Barnes W L, Dereux A and Ebbesen T W 2003 Surface Plasmon Subwavelength Optics Nature 424 824-30

[3] Raether H 1988 Surface Plasmons on Smooth and Rough Surfaces and on Gratings vol 111 of Springer Tracts in Modern Physics (Berlin: SpringerVerlag)

[4] Bohren C F and Huffman D R 1998 Absorption and Scattering of Light by Small Particles (Berlin: Wiley$\mathrm{VCH})$

[5] Homola J 2008 Surface Plasmon Resonance Sensors for Detection of Chemical and Biological Species Chem. Rev. 108 462-93 
[6] Anker J N, Hall W P, Lyandres O, Shah N C, Zhao J and Van Duyne R P 2008 Biosensing with plasmonic nanosensors Nat. Mater. 7 442-53

[7] Ghenuche P, Cherukulappurath S, Taminiau T H, van Hulst N F and Quidant R 2008 Spectroscopic Mode Mapping of Resonant Plasmon Nanoantennas Phys. Rev. Lett. 101116805

[8] Genet C and Ebbesen T W 2007 Light in tiny holes Nature 445 39-46

[9] Ozbay E 2006 Plasmonics: Merging Photonics and Electronics at Nanoscale Dimensions Science 311 18993

[10] Nikolajsen T, Leosson K and Bozhevolnyi S I 2004 Surface Plasmon Polariton Based Modulators and Switches Operating at Telecom Wavelengths Appl. Phys. Lett. 85 5833-5

[11] Krasavin A V, MacDonald K F, Zheludev N I and Zayats A V 2004 High-contrast modulation of light with light by control of surface plasmon polariton wave coupling Appl. Phys. Lett. 85 3369-71

[12] Dicken M J, Sweatlock L A, Pacifici D, Lezec H J, Bhattacharya K and Atwater H A 2008 Electrooptic Modulation in Thin Film Barium Titanate Plasmonic Interferometers Nano Lett. 8 4048-52

[13] Gómez-Rivas J, Sánchez-Gil J A, Kuttge M, Bolivar P H and Kurz1 H 2006 Optically switchable mirrors for surface plasmon polaritons propagating on semiconductor surfaces Phys. Rev. B 74245324

[14] Pacifici D, Lezec H J and Atwater H A 2007 All-optical modulation by plasmonic excitation of CdSe quantum dots Nat. Photonics $1402-6$

[15] Wallis R F, Brion J J, Burstein E and Hartstein A 1974 Theory of surface polaritons in anisotropic dielectric media with application to surface magnetoplasmons in semiconductors Phys. Rev. B 9 3424-37

[16] Abe M and Suwa T 2004 Surface plasma resonance and magneto-optical enhancement in composites containing multicore-shell structured nanoparticles Phys. Rev. B 70235103

[17] Diwekar M, Kamaev V, Shi J and Vardeny Z V 2004 Optical and magneto-optical studies of twodimensional metallodielectric photonic crystals on cobalt films Appl. Phys. Lett. 84 3112-4

[18] Ctistis G, Papaioannou E, Patoka P, Gutek J, Fumagalli P, and Giersig M 2009 Optical and Magnetic Properties of Hexagonal Arrays of Subwavelength Holes in Optically Thin Cobalt Films Nano Lett. 9 1-6

[19] Clavero C, Armelles G, Margueritat J, Gonzalo J, del Muro M G, Labarta A and Batlle X 2007 Interface effects in the magneto-optical properties of Co nanoparticles in dielectric matrix Appl. Phys. Lett. 90 182506

[20] Melle S, Menéndez J L, Armelles G, Navas D, Vázquez M, Nielsch K, Wehrspohn R B and Gösele U 2003 Magneto-optical properties of nickel nanowire arrays Appl. Phys. Lett. 83 4547-9

[21] González-Díaz J, García-Martín A, Armelles G, Navas D, Vázquez M, Nielsch K, Wehrspohn R and Gösele U 2007 Enhanced Magneto-Optics and Size Effects in Ferromagnetic Nanowire Arrays Adv. Mater. $192643-7$

[22] González-Díaz J B, García-Martín A, García-Martín J M, Cebollada A, Armelles G, Sepúlveda B, Alaverdyan Y and Käll M 2008 Plasmonic Au/Co/Au Nanosandwiches with Enhanced Magneto-optical Activity Small 4 202-5

[23] Armelles G, González-Díaz J B, García-Martín A, García-Martín J M, Cebollada A, González M U, Acimovic S, Cesario J, Quidant R and Badenes G 2008 Localized surface plasmon resonance effects on the magneto-optical activity of continuous Au/Co/Au trilayers Opt. Express 16 16104-12

[24] González-Díaz J B, García-Martín A, Armelles G, García-Martín J M, Clavero C, Cebollada A, Lukaszew R A, Skuza J R, Kumah D P and Clarke R 2007 Surface-magnetoplasmon nonreciprocity effects in noblemetal/ferromagnetic heterostructures Phys. Rev. B 76153402

[25] Ferreiro Vila E, Bendaña X M, González-Díaz J B, García-Martín A, García-Martín JM, Cebollada A, Armelles G, Meneses D, Muñoz Sandoval E 2008 Surface plasmon resonance effects in the magnetooptical activity of Ag/Co/Ag trilayers IEEE Trans. Magn. 44 3303-6

[26] Hulteen J C and Van Duyne R P 1995 Nanosphere lithography: A materials general fabrication process for periodic particle array surfaces J. Vac. Sci. Technol. A $131553-8$

[27] Hanarp P, Sutherland D S, Gold J and Kasemo B 2003 Control of nanoparticle film structure for colloidal lithography Colloids Surf. A 214 23-36

[28] Hanarp P, Käll M and Sutherland D S 2003 Optical Properties of Short Range Ordered Arrays of Nanometer Gold Disks Prepared by Colloidal Lithography J. Phys. Chem. B 107 5768-72

[29] Kim W S, Aderholz M and Kleemann W 1993 Calibration of polar Kerr rotation and ellipticity measurements Meas. Sci. Technol. 4 1275-80

[30] García-Martín A, Armelles G and Pereira S 2005 Light transport in photonic crystals composed of magneto-optically active materials Phys. Rev. B 71205116 
[31] Balcells L, Martinez B, Iglesias O, Garcia-Martin J M, Cebollada A, Garcia-Martin A, Armelles G, Sepúlveda B and Alaverdyan Y 2009 Exchange bias in laterally oxidized Au/Co/Au nanopillars Appl. Phys. Lett. 94062502

[32] Hermann C, Kosobukin V A, Lampel G, Peretti J, Safarov V I, and Bertrand P 2001 Surface-enhanced magneto-optics in metallic multilayer films Phys. Rev. B 64235422

[33] Kumah D P, Cebollada A, Clavero C, García-Martín J M, Skuza J R, Lukaszew R A and Clarke R 2007 Optimizing the planar structure of (111) Au/Co/Au trilayers J. Phys. D: Appl. Phys. 40 2699-704

[34] Sepúlveda B, Calle A, Lechuga L M and Armelles G 2006 Highly sensitive detection of biomolecules with the magneto-optic surface-plasmon-resonance sensor Opt. Lett. 31 1085-7 